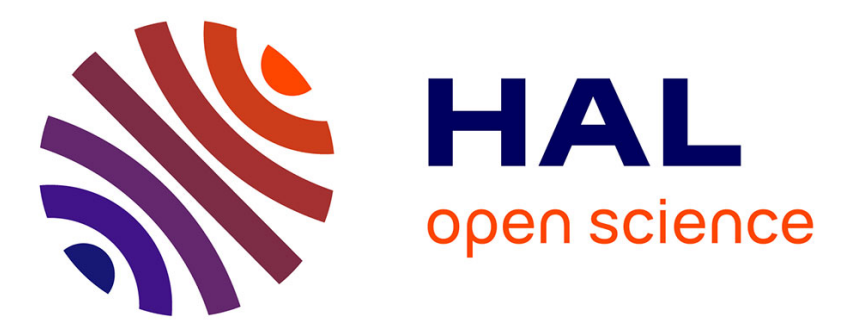

\title{
Déconstruire les idées reçues sur les start-up, la nouvelle responsabilité dévolue aux acteurs de l'accompagnement entrepreneurial
}

\author{
Thomas Houy, Flavien Bazenet
}

\section{- To cite this version:}

Thomas Houy, Flavien Bazenet. Déconstruire les idées reçues sur les start-up, la nouvelle responsabilité dévolue aux acteurs de l'accompagnement entrepreneurial. Entreprendre \& Innover, 2020, L'impact du numérique dans l'éducation et l'accompagnement entrepreneurial, 47, pp.39-50. 10.3917/entin.047.0039 . hal-03541792

\section{HAL Id: hal-03541792 \\ https://hal.science/hal-03541792}

Submitted on 20 Feb 2022

HAL is a multi-disciplinary open access archive for the deposit and dissemination of scientific research documents, whether they are published or not. The documents may come from teaching and research institutions in France or abroad, or from public or private research centers.
L'archive ouverte pluridisciplinaire HAL, est destinée au dépôt et à la diffusion de documents scientifiques de niveau recherche, publiés ou non, émanant des établissements d'enseignement et de recherche français ou étrangers, des laboratoires publics ou privés. 


\section{LA NOUVELLE RESPONSABILITE DEVOLUE AUX ACTEURS DE L'ACCOMPAGNEMENT ENTREPRENEURIAL : DECONSTRUIRE LES IDEES REÇUES SUR LES START-UPS}




\section{Article}

\section{Titre}

La nouvelle responsabilité dévolue aux acteurs de l'accompagnement entrepreneurial : déconstruire les idées reçues sur les start-ups

\section{Auteurs}

Bazenet Flavien et Houy Thomas

Parues dans

Entreprendre \& Innover

\section{Année de publication}

2021

\section{Référence complète}

Houy, T. \& Bazenet, F. (2020). Déconstruire les idées reçues sur les start-up, la nouvelle responsabilité dévolue aux acteurs de l'accompagnement entrepreneurial. Entreprendre \& Innover, 4(4), 39-50 


\title{
La nouvelle responsabilité dévolue aux acteurs de l'accompagnement entrepreneurial : déconstruire les idées reçues sur les start-ups ${ }^{1}$
}

\begin{abstract}
Résumé
L'intense médiatisation autour des start-ups du numérique a conduit à populariser, auprès du grand public, un certain nombre d'idées reçues sur l'entrepreneuriat. Le présent article établit que les primo-entrepreneurs, en raison de leur manque d'expérience dans la conduite d'un projet de création d'entreprise, adhérent à ces lieux communs. Les résultats de cette recherche confèrent donc aux acteurs de l'accompagnement entrepreneurial une nouvelle prérogative : déconstruire, auprès des jeunes porteurs de projets, l'ensemble de ces clichés et de ces représentations fallacieuses du monde des start-ups véhiculés par la presse.

L'article se fonde sur des données d'enquêtes réalisées auprès de 90278 personnes ayant signalé leur intérêt pour la création d'entreprise entre 2007 et 2018. Nous isolons les réponses données par les porteurs de projets numériques $(n=1425)$ et les comparons à celles des autres répondants ( $n=88$ 853). L'examen des croyances de ce large panel de répondants permet de montrer que les primo-entrepreneurs du numérique pensent pouvoir accéder à des rémunérations élevées, envisagent le succès avec une probabilité forte, se lancent dans l'entrepreneuriat en étant relativement peu préparés et anticipent une amélioration de leur employabilité en cas d'échec de leur entreprise.
\end{abstract}

\section{Mots clefs}

Accompagnement entrepreneurial, entrepreneuriat numérique, mythes.

\footnotetext{
${ }^{1}$ Les auteurs souhaitent remercier chaleureusement l'association « CCI - Entreprendre en France » et les Chambres de Commerce et d'Industrie qui ont créé l'Observatoire Permanent des Porteurs de Projet. Elles trouveront ici des remerciements sincères pour avoir accepté de partager l'accès à leurs données. Les auteurs assument seuls la responsabilité des propos et de l'analyse conduite dans l'article.
} 


\section{Points clés de l'article et implications pour les praticiens}

- Cet article vise à informer les acteurs de l'accompagnement entrepreneurial des croyances et de l'état d'esprit de la nouvelle génération d'entrepreneurs du numérique. Cette étude établit que les jeunes porteurs de projet adhérent désormais à un certain nombre d'idées fausses sur l'entrepreneuriat digital. II revient donc aux acteurs de l'accompagnement de prendre connaissance de ces mythes, largement répandus dans la communauté des primo-entrepreneurs, de manière à déconstruire ces clichés et ainsi renforcer l'efficacité de leurs actions d'appui et de soutien aux entrepreneurs.

- Les primo-entrepreneurs du numérique montrent une confiance exagérée dans le succès de leurs start-ups. Cette surévaluation des probabilités de succès peut les amener à une moindre résilience. Il revient donc aux acteurs de l'accompagnement entrepreneurial de tempérer les projections de ces entrepreneurs, de manière à améliorer leur résistance et leur endurance. Cette recommandation souffre de quelques exceptions, notamment dans les cas où l'enthousiasme du fondateur est l'un des fondements de son engagement.

- L'une des nouvelles prérogatives à la charge des accompagnateurs de start-ups consiste à faire en sorte que les entrepreneurs rompent avec le mythe de l'épiphanie entrepreneuriale. Cette idée reçue est préjudiciable aux entrepreneurs en ce qu'elle les conduit à une impréparation fâcheuse et néfaste pour leur projet.

- Les primo-entrepreneurs ont une mauvaise appréciation des opportunités auxquelles ils pourront prétendre à l'issue de leur aventure entrepreneuriale. Les acteurs de l'accompagnement entrepreneurial doivent donc porter une attention particulière à ce que cette erreur d'analyse des entrepreneurs ne se traduise pas par une forme de désinvolture ou, au contraire, de prudence déraisonnable dans le pilotage de leur projet. 


\section{Introduction}

L'entrepreneuriat digital connaît une période d'intense médiatisation. Par-delà la multiplication des sites spécialisés relatant l'actualité des start-ups sur Internet, les médias traditionnels et les médias grand public consacrent désormais une part importante de leur offre éditoriale aux entrepreneurs du Web.

En témoigne le choix en janvier 2020 de la chaîne de télévision M6 de retransmettre en prime time l'émission « Qui veut être mon associé ? ». Ce programme, exclusivement consacré aux start-ups, met en scène des entrepreneurs en situation de levée de fonds. II y a quelques années, le sujet abordé n'aurait certainement pas attiré le grand public. Pourtant, aujourd'hui, l'une des chaînes les plus regardées du Paysage Audiovisuel Français assume d'offrir à cette émission la place la plus convoitée de sa grille : le prime time. Ce choix, s'il en était besoin, atteste désormais que l'entrepreneuriat est devenu un sujet médiatique majeur, susceptible d'attirer une très large audience.

Cet engovement des médias pour l'entrepreneuriat devrait, en principe, être une aubaine. II est au contraire devenu problématique en raison des nombreuses approximations commises par les journalistes pour décrire le monde des start-ups.

Dans un article ${ }^{2}$ publié en 2019, nous avons révélé que la presse véhicule un certain nombre d'idées fausses sur l'entrepreneuriat numérique. Plus précisément, nous montrons que les journalistes opèrent des raccourcis et défendent une image de l'entrepreneuriat éloignée de celle qui résulte des travaux de chercheurs en Sciences de Gestion. Autrement dit, le traitement médiatique sur les start-ups tend à créer une forme de mythologie, en grande partie inexacte, sur la création et le développement des jeunes entreprises du numérique.

Dans ce contexte, il convient de savoir si ce discours médiatique sur l'entrepreneuriat, pénétré par de nombreuses approximations, percole auprès des porteurs de projets. Sans

\footnotetext{
${ }^{2}$ Bazenet F. et Houy T., (2019), "Le paradigme médiatique sur l'entrepreneuriat numérique”, Entreprendre \& Innover, Vol.1, n 40 , pp. 8-21
} 
dévoiler ici, dès l'introduction, l'ensemble des résultats de l'article, nous pouvons révéler que les jeunes entrepreneurs développent malheureusement une perception de l'entrepreneuriat numérique conforme à l'idéal-type proposé par les médias. Il devient donc indispensable d'en tirer une série de recommandations pour orienter le travail des acteurs de l'accompagnement entrepreneurial.

L'intention du présent article est ainsi établie. Dans la présente étude, notre problématique de recherche consiste à évaluer le niveau d'adhésion des porteurs de projets au récit médiatique sur l'entrepreneuriat de manière à en tirer des préconisations pour orienter le travail des acteurs de l'accompagnement entrepreneurial.

Avant cette étude, aucune recherche à notre connaissance n'avait encore établi si l'effet de mode entrepreneurial observé ces dernières années avait eu pour conséquence d'amener à l'entrepreneuriat des créateurs d'entreprise d'un nouveau genre. En offrant une description détaillée de l'état d'esprit des primo-entrepreneurs digitaux, cet article invite les formateurs et les accompagnateurs à ajuster le contenu de leur accompagnement. II leur suggère en particulier d'intensifier leurs efforts de déconstruction des représentations inexactes développées par les jeunes entrepreneurs.

Sur un plan académique, l'article ambitionne de compléter la littérature scientifique en Sciences de Gestion à laquelle peuvent accéder les acteurs de l'accompagnement entrepreneurial. De par leur position, les accompagnateurs sont souvent intéressés par les travaux en lien avec les notions d'intention entrepreneuriale ${ }^{3}$ et de facteurs clefs de succès ${ }^{4}$. Puisqu'ils cherchent à renforcer la qualité de leurs conseils aux entrepreneurs, ils souhaitent en effet accéder à l'état de l'art de ce qui se dit sur les variables susceptibles d'impacter d'une part la motivation d'un porteur de projet à entreprendre et d'autre part la réussite de son projet.

\footnotetext{
${ }^{3}$ Krueger N.F. (1993), "The impact of prior entrepreneurial exposure on perceptions of new venture feasibility and desirability", Entrepreneurship Theory \& Practice, Vol. 18, n¹, pp. 5-20.

${ }^{4}$ Omrane A., Fayolle A., Zeribi-Benslimane O. (2011), "Les compétences entrepreneuriales et le processus entrepreneurial : une approche dynamique”, Revue des Sciences de Gestion, Direction et Gestion, n²51, pp. 91100.
} 
Cet intérêt des accompagnateurs pour cette littérature $n^{\prime}$ est pas neutre ou sans effet puisque les accompagnateurs eux-mêmes exercent une influence sur l'intention entrepreneuriale et les probabilités de succès des entreprises nouvellement créées. Plusieurs publications en ont fait la démonstration ${ }^{5}$. La présente étude s'inscrit donc dans la lignée de ces travaux et participe de l'effort de recherche pour aider les accompagnateurs à percevoir leur rôle et leur impact auprès des entrepreneurs.

Deux champs de la littérature en Sciences de Gestion sont en lien étroit avec le sujet abordé. Le premier est composé de l'ensemble des travaux gestionnaires sur l'intention entrepreneuriale. Les recherches empiriques sur ce thème ont montré que l'intention entrepreneuriale pouvait dépendre de la propension du porteur de projet à vouloir prendre des risques ${ }^{6}$, de son expérience professionnelle ${ }^{7}$, du suivi préalable d'une formation à I'entrepreneuriat $^{8}$, de son envie de voir son revenu augmenter ${ }^{9}$ mais également de son sex $\mathrm{e}^{10}$, de son âge ${ }^{11}$ et de son niveau de diplôme ${ }^{12}$. D'autres auteurs se sont saisis du sujet de manière plus conceptuelle en proposant une modélisation de l'intention entrepreneuriale ${ }^{13}$. Ils

\footnotetext{
${ }^{5}$ Boissin J., Chollet B. et Emin S. (2009), "Les déterminants de l'intention de créer une entreprise chez les étudiants : un test empirique", M@n@gement, Vol.12,n¹, pp. 28-51.

Ferry A. (2018), "L'accompagnement entrepreneurial : la métamorphose des accompagnateurs en facilitateurs". Thèse de doctorat.

Tounés A. (2006), "L'intention entrepreneuriale des étudiants : le cas français", La Revue des Sciences de Gestion, Vol.219, n³, pp.57-65.

Valéau, P. (2006), "L'accompagnement des entrepreneurs durant les périodes de doute", Revue de l’Entrepreneuriat, Vol. 5, n¹, pp. 31-57.

${ }^{6}$ Estay C., Durrieu F. et Akhter M. (2013), "Entrepreneurship: From motivation to start-up”, Journal of International Entrepreneurship, Vol. 11, n³, pp. 243-267.

${ }^{7}$ Siu W. et Lo E. (2013), "Cultural Contingency in the Cognitive Model of Entrepreneurial Intention", Entrepreneurship: Theory \& Practice, Vol. 37, n², pp. 147-173.

${ }^{8}$ Raposo B., Ferreira M., Finisterra do Paço A. et Rodrigues G., (2008), "Propensity to firm creation: empirical research using structural equations", International Entrepreneurship and Management Journal, Vol. 4, nº 4, pp. 485-504.

${ }^{9}$ Benzing C., Chu H. et Kara O. (2009), "Entrepreneurs in Turkey: a factor analysis of motivations, success factors, and problems", Journal of Small Business Management, Vol. 47, n 1, pp. 58-91.

${ }^{10}$ Johansen V. et Foss L. (2013), “The effects of entrepreneurship education - does gender matter?", International Journal of Entrepreneurship and Small Business, Vol.20, n³, pp.255-271.

${ }^{11}$ Tornikoski E.T., Kautonen T. et Le Loarne S. (2012), "Le rôle de l'âge dans l'intention entrepreneuriale : Quelles leçons sur les seniors ?”, Revue Française de Gestion, Vol . 8, n²27, pp. 95-109.

${ }^{12}$ Franke N. et Lüthje C. (2004), "Entrepreneurial intentions of business students-A benchmarking study", International Journal of Innovation and Technology Management, Vol. 1, n³ 3, pp. 269-288.

${ }^{13}$ Shapero A. et Sokol L. (1982), The social dimension of entrepreneurship, The Encyclopedia of Entrepreneurship, Prentice Hall. Krueger N.F. (1993), "The impact of prior entrepreneurial exposure on perceptions of new venture feasibility and desirability", Entrepreneurship Theory \& Practice, Vol. 18, n ${ }^{\circ}$, pp. 520.
} 
défendent alors l'idée selon laquelle l'acte entrepreneurial peut être affecté par plusieurs variables $^{14}$ : le désir de créer du porteur de projet, sa perception des normes sociales, sa propension à l'action et la faisabilité du projet.

Les articles sur l'intention entrepreneuriale visent cependant à identifier les raisons de l'action entrepreneuriale de créateurs d'entreprise, le plus souvent ayant déjà entrepris. Notre étude vise à appréhender la perception de l'écosystème entrepreneurial développée par des personnes qui expriment le souhait d'entreprendre dans un futur proche. Par conséquent, l'objectif du présent article n'est pas de qualifier une intention entrepreneuriale. L'imaginaire discuté dans l'article porte ainsi sur un périmètre plus large et plus amont que I'intention entrepreneuriale, notamment en intégrant des dimensions d'appréciation du contexte entrepreneurial. Dans les termes du modèle $d$ 'intention entrepreneuriale proposé par Krueger ${ }^{15}$, la présente étude peut être considérée comme un travail de qualification des variables perçues de désirabilité, de normes sociales et de contrôle comportemental.

Le deuxième champ de la littérature concerné par notre étude se compose des travaux ayant traité des facteurs clefs de succès des start-ups Internet. Ces travaux nous permettent de rapprocher l'imaginaire des futurs entrepreneurs avec des faits avérés sur ce qui fonde le succès des entreprises sur Internet. Une partie des articles publiés aborde le sujet de manière empirique. A l'aide de bases de données sur la performance, l'environnement, les caractéristiques et les actions d'un certain nombre d'entreprises innovantes, des chercheurs identifient par régression l'ensemble des variables susceptibles d'influencer significativement leur probabilité de succès et/ou de survie. Parmi les facteurs clefs recensés figurent le montant du capital investi et la structure de la clientèle ${ }^{16}$, l'expérience et le niveau de qualification du fondateur ${ }^{17}$, la préparation du projet ${ }^{18}$, l'aptitude du fondateur à apprendre

\footnotetext{
${ }^{14}$ Fayolle A., Gailly B. et Lassas-Clerc N. (2006), "Assessing the impact of entrepreneurship education programmes: a new methodology”, Journal of European Industrial Training, Vol. 30, n9, pp. 701-720.

${ }^{15}$ Krueger N.F. (1993), “The impact of prior entrepreneurial exposure on perceptions of new venture feasibility and desirability", Entrepreneurship Theory \& Practice, Vol. 18, $n^{\circ} 1$, pp. 5-20.

${ }^{16}$ Lasch F., Le roy F. et Yami S. (2005), "Les déterminants de la survie et de la croissance des start-up TIC", Revue Française de Gestion, Vol. 2, n¹55, pp. 37-56.

${ }^{17}$ Barringer B.R., Jones F.F. et Neubaum D.O. (2005), “A quantitative content analysis of the characteristics of rapid-growth firms and their founders", Journal of Business Venturing, Vol.20, $n^{\circ} 5$, pp. 663-687.

${ }^{18}$ Hansen E. L. (1995), "Entrepreneurial networks and organizational growth", Entrepreneurship Theory \& Practice, pp. 7-19.
} 
et la capacité de la start-up à progresser par paliers ou à pivoter ${ }^{19}$. D'autres publications adoptent une approche plus normative, fondée sur des cas particuliers d'entreprise ou des représentations théoriques. Ces travaux portent par exemple des recommandations sur les compétences requises par les entrepreneurs à chaque étape du processus entrepreneurial ${ }^{20}$. Ils discutent également de l'impact des qualités intrinsèques de leader du fondateur ${ }^{21}$ ou encore des bonnes pratiques marketing et de conception à adopter avec ses clients ${ }^{22}$. Certains travaux enfin, sont hybrides. Ils reposent sur une base de données dont le traitement permet de questionner une pratique, un mode de raisonnement ou la pertinence d'une $\operatorname{action}^{23}$

\section{Méthodologie}

Nos résultats proviennent de traitements statistiques opérés sur des données d'enquêtes détenues par l'Observatoire Permanent des Porteurs de Projet. Tous les ans, les Chambres de Commerces et d'Industrie $(\mathrm{CCl})$ de toutes les régions de France réalisent une enquête auprès des personnes qui se sont déplacées chez elles dans le but de faire progresser leur projet entrepreneurial. Ces personnes sont donc des porteurs de projets entrepreneuriaux n'ayant pas encore créé leur entreprise.

L'enquête réalisée par la CCl prend la forme d'un questionnaire comprenant une trentaine de questions fermées. Pour les besoins de notre étude, nous avons eu accès aux réponses

\footnotetext{
${ }^{19}$ Marmer M. et Dogrultan E. (2012), A new framework for understanding why startups succeed, Startup Genome Report.

${ }^{20}$ Omrane A., Fayolle A., Zeribi-Benslimane O. (2011), "Les compétences entrepreneuriales et le processus entrepreneurial : une approche dynamique”, Revue des Sciences de Gestion, Direction et Gestion, $n^{\circ} 251$, pp. 91 100.

${ }^{21}$ Muzyka D., Birley S. et Leleux B. (1995), “Trade-off in the investment decisions of European venture capitalists”, Journal of Business Venturing, Vol. 11, pp. 273-287.

${ }^{22}$ Von Hippel E. (1986), "Lead users: An important source of novel product concepts", Management Science, Vol.32, $n^{\circ} 7$, pp.791-805.

${ }^{23}$ Sarasvathy, S. (2001), "Causation and effectuation: Toward a theoretical shift from economic inevitability to entrepreneurial contingency”, Academy of Management Review, Vol. 26., n², pp. 243-263. Sarasvathy S. (2004) What makes entrepreneurs entrepreneurial?, Batten Briefings Summer 2004.
} 
données à ce questionnaire par 90278 porteurs de projets entrepreneuriaux entre 2007 et 2018.

Pour traiter notre question de recherche, un groupe particulier de porteurs de projets a fait l'objet de traitements statistiques dédiés. Il s'agit des porteurs de projets numériques $(n=1$ 425). II regroupe l'ensemble des personnes ayant déclaré vouloir créer une entreprise dans les secteurs du e-commerce, des services informatiques et du développement de logiciels, de la conception multimédia, des agences Web et de la publicité en ligne. Dans la suite de l'article, nos analyses se fondent sur des comparaisons entre les réponses données par cette catégorie spécifique de porteurs de projets $(n=1425)$ et par les autres répondants à l'enquête $(n=88853)$.

Les caractéristiques des porteurs de projets, et plus spécifiquement de ceux qui ont un projet numérique, sont données dans le tableau 1.

\begin{tabular}{|c|c|c|}
\cline { 2 - 3 } \multicolumn{1}{c|}{} & Porteurs de projets non digitaux & Porteurs de projets digitaux \\
\hline Nombre & 88853 personnes & 1425 personnes \\
\hline Pourcentage de femmes & $43 \%$ & $42 \%$ \\
\hline Âge moyen & 36 ans et 10 mois & 35 ans et 10 mois \\
\hline $\begin{array}{c}\text { Pourcentage de personnes ayant } \\
\text { un diplôme supérieur ou égal à } \\
\text { Bac+2 }\end{array}$ & $42 \%$ & $56 \%$ \\
\hline $\begin{array}{c}\text { Pourcentage de personnes } \\
\text { percevant des allocations } \\
\text { chômage }\end{array}$ & $39 \%$ & $44 \%$ \\
\hline $\begin{array}{c}\text { Pourcentage de personnes } \\
\text { exerçant une activité } \\
\text { professionnelle }\end{array}$ & $43 \%$ & $34 \%$ \\
\hline
\end{tabular}

Tableau 1 : caractéristiques des porteurs de projets entrepreneuriaux considérés dans l'article 
Les données d'enquête sur lesquelles nous opérons nos traitements statistiques montrent un avantage et un inconvénient. L'avantage indéniable de notre base de données concerne le nombre de répondants. La taille de notre échantillon (90 278 porteurs de projets dont 1425 porteurs de projets numériques) garantit la représentativité de nos résultats statistiques, relativement à la population considérée. Le nombre de répondants étant particulièrement élevé, nos manipulations sur les données ne sont en effet pas limitées par les habituelles, mais néanmoins fâcheuses, contraintes statistiques imputables à la sous-représentation d'une catégorie d'acteurs. Pour autant, l'accès à ce large panel de porteurs de projets est rendu possible au prix d'une qualité dégradée des données. Les répondants aux enquêtes proposées par les $\mathrm{CCl}$ sont indifféremment des porteurs de projets engagés ou de simples curieux dont I'intention entrepreneuriale peut être questionnée. II nous est malheureusement impossible de discriminer le niveau d'engagement des répondants sur leurs projets. Aucune variable ni aucun proxy ne permet d'accéder à ce genre d'information.

Tous les arguments avancés dans l'article doivent donc être compris à la lumière de cette limite importante concernant la qualité de notre base de données. Pour cette raison, nos résultats doivent sans doute être envisagés comme des hypothèses ou des tendances dont nous nous saisissons pour ouvrir des discussions. Pour vérifier la validité de ces hypothèses ou de ces tendances, il conviendrait de compléter la présente étude par d'autres travaux, fondés sur des données plus robustes ou adoptant une approche plus qualitative.

\section{Résultats}

L'examen des données d'enquête nous permet d'avancer une thèse : les porteurs de projets digitaux ont une perception singulière de l'entrepreneuriat. Relativement aux autres porteurs de projets, ils montrent davantage d'optimisme et de confiance dans leur entreprise en préparation. A de nombreux égards, leur perception de l'entrepreneuriat rejoint la mythologie entrepreneuriale proposée par les médias généralistes.

\section{Le mythe de l'argent et du succès faciles sur Internet}


Lorsque les médias traitent de l'actualité des levées de fonds et des introductions en bourse des start-ups les plus prometteuses, ils rendent légitimement compte de succès fulgurants. Cette exposition médiatique laisse croire aux personnes extérieures à l'écosystème entrepreneurial que les succès sur Internet sont relativement faciles à obtenir. Pourtant les échecs sont particulièrement nombreux. Certaines études évaluent à $39 \%$ le taux de survie après 5 ans des start-ups du secteur des Technologies de l'Information et de la Communication, soit 10 points de moins que dans les autres secteurs d'activité ${ }^{24}$.

\begin{tabular}{|c|c|c|c|c|}
\hline & \multirow{2}{*}{$\begin{array}{l}\text { Porteurs de projets } \\
\text { non digitaux }\end{array}$} & \multicolumn{3}{|c|}{ Porteurs de projets digitaux* } \\
\hline & & E-commerce & $\begin{array}{c}\text { Services info. et } \\
\text { dev. logiciels }\end{array}$ & $\begin{array}{l}\text { Conception, } \\
\text { agences, pub. }\end{array}$ \\
\hline $\begin{array}{l}\text { \% de personnes déclarant anticiper une } \\
\text { augmentation "très élevée" ou "élevée" de } \\
\text { leur revenu suite à la création de leur } \\
\text { entreprise }\end{array}$ & $54 \%$ & $56 \%$ & $46 \%$ & $75 \%$ \\
\hline $\begin{array}{l}\text { \% de personnes se dèclarant "extrêmement } \\
\text { motivèes" pour entreprendre (les autres se } \\
\text { déclarant simplement "motivées") }\end{array}$ & $77 \%$ & $81 \%$ & $\begin{array}{l}67 \% \\
75 \%\end{array}$ & $66 \%$ \\
\hline $\begin{array}{c}\text { \% de personnes ayant déjà exercé dans le } \\
\text { secteur d'activité vers lequel elles } \\
\text { s'engagent }\end{array}$ & $51 \%$ & $36 \%$ & $74 \%$ & $75 \%$ \\
\hline
\end{tabular}

Tableau 2 : comparaison de certaines réponses données par l'ensemble des porteurs de projets et par les porteurs de projets digitaux.

* Les projets digitaux sont ventilés par type : projets de e-commerce, projets de services informatiques et développement logiciel, projets de conception multimédia, agences Web et publicité en ligne.

Les données d'enquête (tableau 2) montrent que les futurs entrepreneurs du Web expriment une grande confiance sur le niveau de revenu auquel ils accéderont grâce à leur activité à venir

\footnotetext{
${ }^{24}$ Lasch F., Le roy F. et Yami S. (2005), “Les déterminants de la survie et de la croissance des start-up TIC”, Revue Française de Gestion, Vol. 2, n¹55, pp. 37-56.
} 
relativement aux autres porteurs de projets. Par exemple, les porteurs de projets de ecommerce sont $56 \%$ à anticiper une augmentation « très élevée » ou « élevée » de leur salaire actuel. Dans les secteurs d'activité hors digital, ils sont $54 \%$ à partager ce sentiment. L'optimisme des primo-entrepreneurs sur leurs revenus futurs n'est donc pas réservé aux seuls porteurs de projets digitaux et s'observe au sein de l'ensemble de la communauté, quel que soit le secteur d'activité visé par le créateur d'entreprise.

La perception des porteurs de projets digitaux semble excessivement optimiste au regard des faits observés sur le niveau moyen de rémunération des entrepreneurs du Web. Un rapport ${ }^{25}$ proposé par Compass, conduit auprès de 11000 start-ups à travers le monde, avance un niveau de rémunération moyen de 39500 dollars pour un fondateur dont la start-up est en phase de conception ou de prototypage du produit/service proposé. Ce niveau de rémunération est vraisemblablement inférieur aux estimations des porteurs de projets digitaux considérés dans notre panel.

N'ayant pas de données sur les niveaux de rémunération des créateurs d'entreprise non digitaux, nous ne pouvons pas juger si l'optimisme des porteurs de projets traditionnels est raisonnable ou exagéré. Nous décidons donc de concentrer notre attention sur les seules croyances des porteurs de projets digitaux. Les anticipations favorables des porteurs de projets digitaux sur leurs niveaux de rémunération future apparaissent d'autant plus irréalistes qu'elles ne se justifient pas par une détermination supérieure à réussir ou un niveau d'expertise plus élevé. En effet, leur niveau de motivation face à leur projet est sensiblement équivalent à celui des autres porteurs de projets. Précisément, ils sont $75 \%$ à se déclarer " extrêmement motivés " par leur projet, contre une moyenne de $77 \%$ sur les autres secteurs d'activité. Par ailleurs, les porteurs de projets digitaux sont proportionnellement plus nombreux à exercer leur projet dans un secteur qu'ils ne connaissent pas. Cette caractéristique se vérifie notamment pour les porteurs de projets e-commerce puisque $36 \%$ d'entre eux seulement ont déjà eu une expérience dans le e-commerce auparavant.

\footnotetext{
${ }^{25}$ Etude Compass (2014), “73\% of Startup Founders Make \$50,000 Per Year or Less “, disponible sur http://blog.startupcompass.co/
} 
L'écart observé entre la perception des porteurs de projets digitaux et les faits, à la fois sur leurs niveaux de rémunération future et sur les difficultés pour réussir, renvoie à l'existence d'un mythe de l'argent et du succès faciles sur Internet. La diffusion de cette idée reçue auprès de la nouvelle population d'entrepreneurs du Web pourrait engendrer deux conséquences fâcheuses. Premièrement, elle peut conduire à observer un certain nombre de frustrations. En ayant initialement surestimé ses revenus et en ayant sous-estimé les difficultés, une partie des entrepreneurs est en effet susceptible de montrer de la déception, de l'impatience et risque d'arrêter prématurément son activité. Deuxièmement, le mythe de l'argent et du

succès faciles sur Internet peut provoquer des phénomènes d'anti-sélection ${ }^{26}$ sur la population d'entrepreneurs. La croyance de l'argent et du succès faciles peut contribuer à attirer vers l'entrepreneuriat la partie de la population la plus sensible à ces variables. Malheureusement, il n'est pas prouvé que cette partie de la population soit la plus compétente pour entreprendre.

\section{Le mythe de l'épiphanie et du temps accéléré sur Internet}

L'épiphanie entrepreneuriale fait partie intégrante de l'imaginaire associé aux start-ups Internet. Les récits médiatiques entrepreneuriaux commencent souvent par ce moment soudain de compréhension qui met en scène un entrepreneur subitement extralucide face à une découverte ou une situation.

Cette représentation est simpliste car elle repose sur deux hypothèses grossières. La première concerne le processus d'idéation. Une relecture attentive des plus grands succès entrepreneuriaux du Web suffirait à montrer que les idées naissent de manières très différentes. Le cheminement des entrepreneurs est toujours atypique. Les entrepreneurs peuvent occuper indifféremment et successivement des rôles d'entrepreneurs frugaux, d'entrepreneurs opportunistes, d'entrepreneurs "sans le savoir», d'entrepreneurs

\footnotetext{
${ }^{26}$ Tirole J. (1988), The Theory of Industrial Organization, MIT Press.
} 
« copieurs", ou encore d'entrepreneurs pragmatiques ${ }^{27}$. Les entrepreneurs visionnaires ne représentent en réalité qu'une petite partie des entrepreneurs ${ }^{28}$.

La deuxième hypothèse contestable renvoie à la manière dont est décrit le raisonnement des entrepreneurs. Le raisonnement des entrepreneurs apparait « causal » alors même que des recherches récentes en entrepreneuriat ont montré que les entrepreneurs faisaient davantage preuve d'une logique "effectuale ${ }^{29}$. Les entrepreneurs n'ont pas d'objectifs à long terme desquels ils déclinent des actions précises pour trouver le moyen d'atteindre leurs buts initiaux. Les entrepreneurs partent plutôt des ressources en leur possession et envisagent l'ensemble des applications auxquelles ils peuvent prétendre.

Croire en l'épiphanie entrepreneuriale revient à minimiser les vertus du temps de préparation des projets entrepreneuriaux. Si les idées surviennent brusquement aux entrepreneurs et dans leurs versions définitives, alors les actions préparatoires à engager avant la création de son entreprise présentent moins d'utilité et deviennent moins nombreuses. Le mythe de l'épiphanie est donc étroitement lié au mythe du temps accéléré sur Internet.

Plusieurs données d'enquête nous permettent d'avancer l'idée que les porteurs de projets digitaux adhèrent au mythe de l'épiphanie et du temps accéléré sur Internet. D'abord, les futurs entrepreneurs du Web montrent un temps de maturation de leur projet légèrement moins long que celui des projets portés par les autres entrepreneurs (tableau 3). En moyenne, le temps de préparation des porteurs de projets digitaux est 5\% moins long (19 mois contre 20 mois). Sur certains secteurs d'activité digitaux comme le développement logiciels ou les agences Web ou de publicité en ligne, ce temps de préparation peut même être 10\% plus court (18 mois contre 20 mois).

\footnotetext{
${ }^{27}$ Alexandre, L. (2016), "Typologie des entrepreneurs, une approche par le genre", Revue de l'Entrepreneuriat, Vol.15, n³, pp.109-127.

${ }^{28}$ Filion L. J. (1998), "Deux types d'entrepreneurs : l'opérateur et le visionnaire. Conséquences pour l'éducation", Cahier de recherche, $\mathrm{N}^{\circ} 1998-10$, septembre, Ecole des Hautes Etudes Commerciales, Montréal. Filion L. J. (2000), "Typologie des entrepreneurs, est-ce vraiment utile?", In T. Verstraete, Histoire d'entreprendre. Les réalités de l'entrepreneuriat (pp. 251-262), Paris, EMS.

${ }^{29}$ Sarasvathy, S. (2001), "Causation and effectuation: Toward a theoretical shift from economic inevitability to entrepreneurial contingency", Academy of Management Review, Vol. 26., n², pp. 243-263. Et Sarasvathy S. (2004) What makes entrepreneurs entrepreneurial?, Batten Briefings Summer 2004.
} 


\begin{tabular}{|c|c|c|c|c|}
\hline & \multirow{2}{*}{$\begin{array}{l}\text { Porteurs de projets } \\
\text { non digitaux }\end{array}$} & \multicolumn{3}{|c|}{ Porteurs de projets digitaux } \\
\hline & & E-commerce & $\begin{array}{l}\text { Services info. et } \\
\text { dev. logiciels }\end{array}$ & $\begin{array}{l}\text { Conception, } \\
\text { agences, pub. }\end{array}$ \\
\hline $\begin{array}{l}\text { Ancienneté moyenne de la réflexion } \\
\qquad \text { du projet }\end{array}$ & 20 mois & 20 mois & 19 mois & 18 mois \\
\hline $\begin{array}{l}\text { \% de personnes qui pensent que la } \\
\text { création d'entreprise en France est } \\
\text { "difficile" ou "très difficile" }\end{array}$ & $77 \%$ & \multicolumn{3}{|c|}{$85 \%$} \\
\hline
\end{tabular}

Tableau 3 : comparaison de certaines réponses données par l'ensemble des porteurs de projets et les porteurs de projets digitaux.

* les cases non renseignées renvoient à un nombre trop faible de répondants pour avoir une signification statistique.

Toutes les choses n'étant pas égales par ailleurs, le temps de préparation relativement court des projets digitaux pourrait éventuellement s'expliquer par un sentiment partagé de simplicité de la part de leurs porteurs sur la manière de créer une entreprise en France. Mais les porteurs de projets digitaux sont au contraire ceux qui perçoivent le plus de difficultés dans la création de leur entreprise. Ils sont $85 \%$ à considérer la création d'une entreprise «très difficile » ou « difficile ». Ce taux est sensiblement plus élevé que celui observé chez les autres porteurs de projets. Cette observation est donc de nature à renforcer la vraisemblance d'une adhésion des entrepreneurs digitaux aux mythes de l'épiphanie et du temps accéléré sur Internet.

La croyance en une épiphanie entrepreneuriale et un temps accéléré sur Internet présente une conséquence majeure : elle pourrait nous conduire à observer une forme d'impréparation préjudiciable aux porteurs de projets digitaux. Les études de faisabilité et la préparation des projets font partie des facteurs clefs de succès des start-ups identifiés par la littérature en 
Science de Gestion ${ }^{30}$. Ces étapes essentielles de la création d'une entreprise ne doivent donc pas être négligées par des entrepreneurs qui attribueraient, à tort, davantage de valeur à l'intuition qu'à l'analyse.

Notons que cette forme d'impréparation des porteurs de projets digitaux, révélée dans notre étude par la comparaison des temps de maturation des projets portés par les créateurs d'entreprises digitales et non digitales, est en train de disparaître progressivement. Nos données se fondent sur les réponses apportées par les primo-entrepreneurs aux questionnaires distribués par les $\mathrm{CCl}$ entre 2007 et 2018. La première intention de notre recherche n'est pas de repérer des tendances dans les réponses données entre 2007 et 2018 pour en tirer des enseignements sur l'évolution de l'état d'esprit des entrepreneurs depuis une dizaine d'année. Pour autant, nous pouvons signaler que sur ce résultat en particulier, le temps de préparation des projets digitaux était significativement inférieur entre 2007 et 2014 (15 mois), relativement à la période 2014 - 2018 (18 mois minimum). Cette observation met en perspective une tendance à la hausse des temps de maturation des projets portés par les entrepreneurs digitaux. Il devient donc possible d'imaginer que les accompagnateurs auront de moins en moins de difficultés à convaincre les primo-entrepreneurs de bien penser et préparer leur projet avant de se lancer.

\section{Le mythe de l'échec stigmatisant}

Quel que soit le secteur d'activité concerné, l'échec entrepreneurial est souvent présenté par la presse comme stigmatisant. De nombreux articles de presse paraissent régulièrement pour dire combien un entrepreneur en situation d'échec rencontre des difficultés à susciter l'intérêt d'un banquier ou d'un employeur.

Les porteurs de projets sont naturellement inquiets quant à leur niveau d'employabilité après une aventure entrepreneuriale échouée (tableau 4). 62\% d'entre eux seulement déclarent

\footnotetext{
${ }^{30}$ Hansen E. L. (1995), “Entrepreneurial networks and organizational growth”, Entrepreneurship Theory \& Practice, pp. 7-19.
} 
être confiants sur la valorisation par un futur employeur d'un éventuel échec de leur projet. Ce qui signifie en creux que $38 \%$ d'entre eux pensent qu'il leur sera difficile de valoriser leur expérience en cas d'échec. Mais il est important de noter l'existence d'une réelle hétérogénéité des points de vue sur ce sujet puisque les porteurs de projets digitaux sont très loin de partager cette inquiétude. $82 \%$ d'entre eux expriment au contraire une grande confiance sur la manière dont un éventuel échec serait perçu. Ils envisagent donc avec sérénité leur future employabilité.

Ce résultat montre que les porteurs de projets digitaux n'adhèrent pas à la présentation habituellement donnée des entrepreneurs ayant connu l'échec. Peut-être expriment-ils par ce résultat l'assurance que le marché de l'emploi saura bientôt valoriser leur savoir-faire, de plus en plus demandé et actionnable dans les grands groupes au regard du rôle joué par les technologies dans l'ensemble des entreprises. Cette assurance des porteurs de projets digitaux est confirmée par le fait qu'ils sont 32\% à considérer l'entrepreneuriat comme « un moyen de se réaliser ". Ce taux est sensiblement équivalent à celui observé dans les autres secteurs d'activité (40\%).

\begin{tabular}{|c|c|c|c|c|}
\cline { 2 - 5 } \multicolumn{1}{c|}{} & \multirow{2}{*}{$\begin{array}{c}\text { Porteurs de projets } \\
\text { non digitaux }\end{array}$} & \multicolumn{2}{|c|}{ Porteurs de projets digitaux } \\
\cline { 3 - 5 } & & E-commerce & $\begin{array}{c}\text { Services info. } \\
\text { et dev. } \\
\text { logiciels }\end{array}$ & $\begin{array}{c}\text { Conception, } \\
\text { agences, pub. }\end{array}$ \\
\hline $\begin{array}{c}\text { \% de personnes déclarant être } \\
\text { confiant sur la valorisation par un } \\
\text { futur employeur d'un éventuel } \\
\text { échec de leur projet }\end{array}$ & $62 \%$ & $82 \%$ & $82 \%$ & non renseigné* \\
\hline $\begin{array}{c}\text { \% de personnes qui voient dans } \\
\text { leur projet "un moyen de se } \\
\text { réaliser" }\end{array}$ & $40 \%$ & $34 \%$ & $33 \%$ & $23 \% *$ \\
\hline
\end{tabular}

Tableau 4 : comparaison de certaines réponses données par l'ensemble des porteurs de projets et par les porteurs de projets digitaux. 
* les cases non renseignées renvoient à un nombre trop faible de répondants pour avoir une signification statistique.

L'atténuation des craintes associées à un échec entrepreneurial pourrait changer la nature des activités portées par les fondateurs de start-ups Internet. En anticipant une augmentation de leur niveau d'employabilité sur le marché de l'emploi, les entrepreneurs du Web pourraient s'engager dans des aventures entrepreneuriales plus risquées.

\section{Conclusion}

La présente recherche montre que les porteurs de projets digitaux affichent une grande confiance dans leur projet. Leur optimisme se traduit par plusieurs croyances, renvoyant pour certaines d'entre elles à des mythes souvent entretenus par les médias grand public. Les porteurs de projets digitaux pensent pouvoir accéder à des rémunérations élevées, ils envisagent le succès de leur projet avec une probabilité forte, ils se lancent dans l'entrepreneuriat en étant légèrement moins préparés et ils anticipent une amélioration de leur employabilité en cas d'échec de leur entreprise.

Le principal apport de l'article réside dans le caractère actionnable des résultats avancés pour les professionnels de l'accompagnement entrepreneurial. Chaque résultat peut en effet être discuté à l'aune de ses conséquences pour les protagonistes de l'aide et du soutien aux entrepreneurs.

La confiance exagérée des primo-entrepreneurs dans le succès de leurs start-ups peut les conduire à abandonner prématurément leurs projets lorsqu'ils se confronteront aux premières difficultés et les amener à une moindre résilience, alors même que cette capacité à surmonter les obstacles apparaît comme décisive pour mener à bien son projet de création d'entreprise ${ }^{31}$. L'un des rôles des accompagnateurs consiste alors à avertir les jeunes entrepreneurs des nombreuses difficultés à venir et, toutes choses égales par ailleurs, des

\footnotetext{
${ }^{31}$ Bonnard F., Toutain O. et Verzat C. (2018), "Dépasser le mythe de l'entrepreneur super héros", Entreprendre \& Innover, Vol.4, n³9, pp.5-9.
} 
probabilités faibles de succès de chaque projet. En tempérant leurs estimations, l'effet recherché est ici de renforcer la résistance et l'endurance des entrepreneurs. Notons que cette recommandation peut faire débat et doit donc être considérée avec prudence car pour certains entrepreneurs, l'enthousiasme est un fondement de leur engagement. Or, cet enthousiasme peut se nourrir d'une forme d'insouciance exprimée par une représentation faussée des probabilités de succès d'un projet. Rétablir des vérités statistiques peut alors avoir l'effet contraire à celui recherché.

Le cliché selon lequel les idées surviennent miraculeusement, par chance et sans travail, est préjudiciable aux primo-entrepreneurs car cela invite à une moindre préparation de leur projet entrepreneurial. Rompre avec ce mythe de l'épiphanie entrepreneuriale est une nouvelle prérogative à la charge des accompagnateurs de start-ups afin de provoquer dans la population des entrepreneurs une croyance forte sur les effets positifs du travail, de l'expérimentation, des tests, de l'exécution, ... en minimisant leur appréciation du paramètre chance dans leur aventure entrepreneuriale.

Notons que sur le plan académique, la notion d'intention entrepreneuriale est souvent étudiée au prisme de la théorie du comportement planifié proposée par Icek Ajzen ${ }^{32}$. L'acte d'entreprendre dépend alors de trois variables : l'attitude du porteur de projet à l'égard de la création d'une entreprise, sa perception des normes sociales et le contrôle qu'il pense avoir sur la situation. Autrement dit, l'engagement d'un porteur de projet est influencé par la désirabilité qu'il exprime sur le fait d'entreprendre, la manière dont il pense que son environnement va juger sa démarche et sa perception de la faisabilité du projet. Nos résultats relatifs aux mythes de l'argent facile sur Internet et de l'épiphanie entrepreneuriale influencent très directement la troisième variable de ce modèle, qui concerne la perception de la faisabilité du projet. Les accompagnateurs, en déconstruisant ces mythes, doivent donc prendre conscience qu'ils pèseront sur l'intention entrepreneuriale des porteurs de projets.

Enfin, il revient aux accompagnateurs d'informer les entrepreneurs sur les issues auxquelles ils pourront prétendre après leurs aventures entrepreneuriales. Une mauvaise évaluation de

\footnotetext{
32 Ajzen I. (1991), "The theory of planned behavior", Organizational Behavior and Human Decision Processes, Vol.50, pp.179-211.
} 
la part des entrepreneurs, à la hausse ou à la baisse, sur la manière dont leur expérience (réussite ou échec) sera valorisée par le marché du travail peut les conduire à piloter leur entreprise avec désinvolture ou, au contraire avec un excès de prudence. Dans les deux cas, il s'agit d'une distorsion de la réalité qui ne leur sera sans doute pas profitable en ce qu'elle pourrait générer chez eux, à terme, beaucoup de déceptions.

Des travaux ont été produits dans le but d'identifier les compétences dont les entrepreneurs devaient faire preuve tout au long du processus de création d'entreprise ${ }^{33}$. Alain Fayolle, Amina Omrane et Olfa Zeribi Ben-Slimane ont par exemple identifié les compétences requises aux différents stades du développement $d^{\prime} u n$ projet entrepreneurial ${ }^{34}$. Au moment du déclenchement du processus entrepreneurial, ces chercheurs montrent que les entrepreneurs doivent pouvoir maîtriser les informations. Ils doivent être capables de rechercher, collecter, classer et exploiter les informations disponibles pour faire progresser leurs projets et passer de l'intention à l'acte entrepreneurial. L'adhésion d'une grande partie des porteurs de projet aux mythes véhiculés par la presse peut donc être lue comme un défaut de compétence de la part de certains porteurs de projet. Ils doivent absolument acquérir ou développer cette compétence relative à l'évaluation critique des informations disponibles sur l'entrepreneuriat. Cette recommandation formulée aux entrepreneurs trouve une contrepartie pour les accompagnateurs. Lorsque les accompagnateurs aident des porteurs de projets à un stade très précoce de leur aventure entrepreneuriale, sans doute doivent-ils désormais essayer de développer chez eux cette habileté à repérer les approximations et les représentations illusoires nourries par le récit médiatique sur l'entrepreneuriat.

L'article propose des résultats directement actionnables par les accompagnateurs et les formateurs. Pour autant, d'autres acteurs de l'écosystème entrepreneurial peuvent également se saisir de la présente étude pour questionner leurs pratiques professionnelles.

\footnotetext{
${ }^{33}$ Toutain O. et Fayolle A. (2008), "Compétences entrepreneuriales et pratiques d'accompagnement : approche exploratoire et modélisation", In Godefroy Kizabi (Ed.), Entrepreneuriat et accompagnement - Outils, actions et paradigmes nouveaux, Paris : L'Harmattan, pp. 31-72.

${ }^{34}$ Omrane A., Fayolle A. et Ben-Slimane O. (2011), "Les compétences entrepreneuriales et le processus entrepreneurial : une approche dynamique", La Revue des Sciences de Gestion, Vol.251, n5, pp. 91-100.
} 
En premier lieu, les médias. La presse peut certainement faire progresser la qualité des contenus qu'elle diffuse sur les start-ups. Dans cette perspective, un rapprochement entre la sphère médiatique et le monde de la recherche peut être souhaitable. Ce rapprochement peut s'opérer au moyen d'un double mouvement, synchrone, des médias vers les chercheurs mais également des chercheurs vers les médias.

En second lieu, les décideurs politiques. Certaines décisions du régulateur visent à faciliter l'accès à l'entrepreneuriat. Alors que ces actions pourraient, de prime abord, paraître louables, elles peuvent être interrogées à la lumière de certains résultats de l'article. Discutons par exemple des subventions accordées aux jeunes entrepreneurs, le plus souvent par la banque publique d'investissement. Ces subventions ont évidemment un effet positif en ce qu'elles permettent de financer l'exploration de certaines start-ups dont le modèle économique n'est pas encore stabilisé. Néanmoins, ces aides comportent potentiellement trois effets négatifs. Elles créent d'abord une forme d'anti-sélection des porteurs de projets en attirant à l'entrepreneuriat des personnes relativement moins engagées et moins résilientes que celles qui entreprendraient si ces aides n'existaient pas. Ces subventions peuvent donc abaisser le niveau moyen de compétences et d'engagement des entrepreneurs. Elles risquent ensuite de développer chez les porteurs de projets de mauvais réflexes. Au démarrage de leurs activités, les entrepreneurs doivent s'assurer du caractère frugal de toutes leurs actions. Leur accorder de l'argent trop tôt, c'est les autoriser à conduire des actions qui les empêchent de développer la sobriété et l'état d'esprit frugal requis pour surmonter les problèmes à venir dans leurs aventures entrepreneuriales. Enfin, les subventions accordées alimentent plusieurs des mythes mis en lumière dans cet article. L'argent public consacré à l'entrepreneuriat nourrit l'idée selon laquelle il est assez facile de tirer des revenus de la création d'entreprise, ce qui tend à favoriser l'impréparation déjà observée chez les entrepreneurs numériques.

Aucune donnée ne nous permet de préjuger de la force des quatre effets que l'on vient d'évoquer. L'effet positif des aides publiques l'emporte peut-être sur leurs trois effets négatifs. Pour autant, à la lumière de cet article, nous pouvons signaler aux décideurs politiques qu'un redéploiement de l'argent public, des subventions à l'égard des start-ups vers la formation, réduirait mécaniquement les trois effets négatifs imputables au 
financement public de jeunes entreprises. La réduction des subventions permettrait en effet de limiter automatiquement l'anti-sélection des entrepreneurs peu résilients et d'encourager la frugalité des décisions des jeunes entrepreneurs. Quand, dans le même temps, le renforcement des efforts de formation à l'entrepreneuriat permettrait de casser les lieux communs qui alimentent les mythes autour de la création de start-ups. 


\section{Références bibliographiques - Article 2}

Bazenet F. et Houy T., (2019), "Le paradigme médiatique sur l'entrepreneuriat numérique", Entreprendre \& Innover, Vol.1, $\mathrm{n}^{\circ}$ 40, pp. 8-21

Ajzen I. (1991), "The theory of planned behavior", Organizational Behavior and Human Decision Processes, Vol.50, pp.179-211.

Alexandre, L. (2016), "Typologie des entrepreneurs, une approche par le genre", Revue de I’Entrepreneuriat, Vol.15, n³, pp.109-127.

Barringer B.R., Jones F.F. et Neubaum D.O. (2005), "A quantitative content analysis of the characteristics of rapid-growth firms and their founders", Journal of Business Venturing, Vol.20, ${ }^{\circ} 5$, pp. 663-687.

Benzing C., Chu H. et Kara O. (2009), "Entrepreneurs in Turkey: a factor analysis of motivations, success factors, and problems", Journal of Small Business Management, Vol. 47, $\mathrm{n}^{\circ} 1$, pp. 58-91.

Boissin J., Chollet B. et Emin S. (2009), "Les déterminants de l'intention de créer une entreprise chez les étudiants : un test empirique", M@n@gement, Vol.12, nº 1, pp. 28-51.

Bonnard F., Toutain O. et Verzat C. (2018), "Dépasser le mythe de l'entrepreneur super héros", Entreprendre \& Innover, Vol.4, n³9, pp.5-9.

Estay C., Durrieu F. et Akhter M. (2013), "Entrepreneurship: From motivation to start-up", Journal of International Entrepreneurship, Vol. 11, n³ 3 , pp. 243-267.

Fayolle A., Gailly B. et Lassas-Clerc N. (2006), "Assessing the impact of entrepreneurship education programmes: a new methodology", Journal of European Industrial Training, Vol. 30, n9, pp. 701-720.

Ferry A. (2018), "L'accompagnement entrepreneurial : la métamorphose des accompagnateurs en facilitateurs". Thèse de doctorat.

Filion L. J. (1998), "Deux types d'entrepreneurs : I'opérateur et le visionnaire. Conséquences pour l'éducation", Cahier de recherche, Nº 1998-10, septembre, Ecole des Hautes Etudes Commerciales, Montréal.

Filion L. J. (2000), "Typologie des entrepreneurs, est-ce vraiment utile ?", In T. Verstraete, Histoire d'entreprendre. Les réalités de l'entrepreneuriat (pp. 251-262), Paris, EMS.

Franke N. et Lüthje C. (2004), "Entrepreneurial intentions of business students-A benchmarking study", International Journal of Innovation and Technology Management, Vol. $1, \mathrm{n}^{\circ} 3$, pp. 269-288.

Hansen E. L. (1995), "Entrepreneurial networks and organizational growth", Entrepreneurship Theory \& Practice, pp. 7-19.

Hansen E. L. (1995), "Entrepreneurial networks and organizational growth", Entrepreneurship Theory \& Practice, pp. 7-19.

Johansen V. et Foss L. (2013), "The effects of entrepreneurship education - does gender matter?", International Journal of Entrepreneurship and Small Business, Vol.20, n³, pp.255271. 
Krueger N.F. (1993), "The impact of prior entrepreneurial exposure on perceptions of new venture feasibility and desirability", Entrepreneurship Theory \& Practice, Vol. $18, n^{\circ} 1$, pp. 520.

Krueger N.F. (1993), "The impact of prior entrepreneurial exposure on perceptions of new venture feasibility and desirability", Entrepreneurship Theory \& Practice, Vol. 18, nº 1 , pp. 520.

Lasch F., Le roy F. et Yami S. (2005), "Les déterminants de la survie et de la croissance des start-up TIC", Revue Française de Gestion, Vol. 2, n¹55, pp. 37-56.

Lasch F., Le roy F. et Yami S. (2005), "Les déterminants de la survie et de la croissance des start-up TIC", Revue Française de Gestion, Vol. 2, n¹55, pp. 37-56.

Marmer M. et Dogrultan E. (2012), A new framework for understanding why startups succeed, Startup Genome Report.

Muzyka D., Birley S. et Leleux B. (1995), "Trade-off in the investment decisions of European venture capitalists", Journal of Business Venturing, Vol. 11, pp. 273-287.

Omrane A., Fayolle A. et Ben-Slimane O. (2011), "Les compétences entrepreneuriales et le processus entrepreneurial : une approche dynamique", La Revue des Sciences de Gestion, Vol.251, n 5 , pp. 91-100.

Omrane A., Fayolle A., Zeribi-Benslimane O. (2011), "Les compétences entrepreneuriales et le processus entrepreneurial : une approche dynamique", Revue des Sciences de Gestion, Direction et Gestion, n²51, pp. 91-100.

Omrane A., Fayolle A., Zeribi-Benslimane O. (2011), "Les compétences entrepreneuriales et le processus entrepreneurial : une approche dynamique", Revue des Sciences de Gestion, Direction et Gestion, n²51, pp. 91-100.

Raposo B., Ferreira M., Finisterra do Paço A. et Rodrigues G., (2008), "Propensity to firm creation: empirical research using structural equations", International Entrepreneurship and Management Journal, Vol. 4, nº, pp. 485-504.

Sarasvathy, S. (2001), "Causation and effectuation: Toward a theoretical shift from economic inevitability to entrepreneurial contingency", Academy of Management Review, Vol. 26., $\mathrm{n}^{\circ} 2$, pp. 243-263. Et Sarasvathy S. (2004) What makes entrepreneurs entrepreneurial?, Batten Briefings Summer 2004.

Sarasvathy, S. (2001), "Causation and effectuation: Toward a theoretical shift from economic inevitability to entrepreneurial contingency", Academy of Management Review, Vol. 26., $\mathrm{n}^{\circ} 2$, pp. 243-263. Sarasvathy S. (2004) What makes entrepreneurs entrepreneurial?, Batten Briefings Summer 2004.

Shapero A. et Sokol L. (1982), The social dimension of entrepreneurship, The Encyclopedia of Entrepreneurship, Prentice Hall. Krueger N.F. (1993), "The impact of prior entrepreneurial exposure on perceptions of new venture feasibility and desirability", Entrepreneurship Theory \& Practice, Vol. 18, n 1 , pp. 5-20.

Siu W. et Lo E. (2013), "Cultural Contingency in the Cognitive Model of Entrepreneurial Intention", Entrepreneurship: Theory \& Practice, Vol. 37, n², pp. 147-173.

Tirole J. (1988), The Theory of Industrial Organization, MIT Press. 
Tornikoski E.T., Kautonen T. et Le Loarne S. (2012), "Le rôle de l'âge dans l'intention entrepreneuriale : Quelles leçons sur les seniors ?", Revue Française de Gestion, Vol . 8, n²27, pp. 95-109.

Tounés A. (2006), "L'intention entrepreneuriale des étudiants : le cas français", La Revue des Sciences de Gestion, Vol.219, n³, pp.57-65.

Toutain O. et Fayolle A. (2008), "Compétences entrepreneuriales et pratiques d'accompagnement: approche exploratoire et modélisation", In Godefroy Kizabi (Ed.), Entrepreneuriat et accompagnement-Outils, actions et paradigmes nouveaux, Paris: L'Harmattan, pp. 31-72.

Valéau, P. (2006), "L'accompagnement des entrepreneurs durant les périodes de doute", Revue de l'Entrepreneuriat, Vol. 5, nº 1 , pp. 31-57.

Von Hippel E. (1986), "Lead users: An important source of novel product concepts", Management Science, Vol.32, nº 7 pp.791-805. 